\title{
Fatores estressantes em cães: percepção dos tutores
}

\author{
Stressful factors in dogs: owner's perception
}

\author{
Tâmara Duarte Borges ${ }^{1 *}$, Flávia Borges Santos ${ }^{1}$, Daniela Mattos Schlindwein ${ }^{1}$, Luciana do Amaral Gurgel Galeb ${ }^{1}$, \\ Melisa Fructuoso Machado² \\ ${ }^{1}$ Pontifícia Universidade Católica do Paraná (PUCPR), Curitiba, PR, Brasil \\ 2 Universidade Federal do Paraná (UFPR), Curitiba, PR, Brasil
}

\section{Resumo}

Objetivou-se investigar a possível presença de fatores estressores no ambiente de convívio de cães por meio de aplicação de questionário aos tutores. Foi utilizada amostra de conveniência constituída por 50 cães atendidos de forma recorrente no Hospital Veterinário da Universidade Federal de Uberlândia (UFU). Os dados foram coletados por dois veterinários submetidos a treinamento prévio e as respostas transferidas para banco de dados (software EpiInfo-6.04) e apresentadas de forma descritiva. A maioria dos tutores mantinha seus animais dentro de casa $(16 \%, \mathrm{n}=8)$ e no quintal $(22 \%$, $\mathrm{n}=11)$, porém, em $70 \%(\mathrm{n}=35)$ dos casos o ambiente de convivência dos cães passou por transformação nos últimos seis meses. Quanto aos fatores sociais, 16\% (n = 8) tiveram a adição de um novo animal ao convívio e $30 \%(\mathrm{n}=15)$ tiveram o lazer reduzido. Tutores $(64 \%$, $\mathrm{n}=32$ ) relataram repreender seus cães quando estes demonstram comportamentos indesejados, sendo a tentativa de cópula com objetos e pessoas o mais citado, e $46 \%(n=23)$ afirmaram puni-los com agressões diretas. Os cães avaliados têm constante comportamento de medo $(74 \%, \mathrm{n}=37)$ e têm preferido ficar isolados nos últimos dias $(60 \%, \mathrm{n}=30)$. Conclui-se que os tutores de animais podem fornecer informações válidas acerca de possíveis fatores estressantes em cães, auxiliando os médicos veterinários no diagnóstico.

Palavras-chave: Ambiente. Bem-estar animal. Cão. Comportamento animal. Estresse.

\section{Abstract}

The aim of this study was to investigate dog's behaviour changes and the presence of stressors in their living environment, based on a questionnaire applied to owners at anamnesis time. It was used a convenience sample composed by 50 dogs recurrently taken to the veterinary hospital of Universidade Federal de Uberlândia. Data was collected by two veterinarians submitted to previous training. The questionnaire data entered into a database created in EpiInfo-6.04 software and it was described in percentage. Most owners maintain their pets indoors $(16 \%, n=8)$ and in the backyard (22\%, $n=11)$, however, 
in $70 \%$ of the cases home environment has gone through changes in the last six months. Regarding social stressors, another animal was added to the family in $16 \%(n=8)$ of the cases, and 30\% ( $n=15)$ had a reduction in leisure actives. Owners (64\%, $n=32)$ reprehend their dogs when they engage in unwanted behaviours, being the copula with objects or people the most cited, and 46\% ( $n=23)$ affirms to punish the dog with direct aggression. The evaluated dogs have constantly fear behaviour (74\%, $n$ $=37)$ and have preferred to be isolated in the past days $(60 \%, n=30)$. We conclude that owners can provide valid information about stressors in dogs, assisting veterinarian's diagnostic.

Keywords: Environment. Animal welfare. Dog. Animal behavior. Stress.

\section{Introdução}

Todos os animais domésticos têm desenvolvido mecanismos para combater o estresse, uma vez que são notórios os efeitos danosos sobre o indivíduo. Gradualmente, foi aceito que os animais também sofrem da carga de estresse e que desenvolvem patologias similares aos seres humanos quando expostos a situações adversas, podendo sucumbir a doenças, ter atraso no crescimento, prejuízos reprodutivos, distúrbios comportamentais ou, ainda, alterações fisiológicas importantes (Rensis e Scaramuzzi, 2003), o que prejudica diretamente seu bem-estar (Rooney et al., 2009).

Níveis aumentados de estresse afetam diretamente a imunidade do animal, diminuindo a atuação e migração das células linfocitárias e leucocitárias para as áreas inflamatórias (Blecha, 2000), diminuindo temporariamente a resposta inflamatória aguda. Em situações de inflamações crônicas, onde há o prolongamento do estresse, acontece a liberação de glicocorticoides que atuam de forma destrutiva nos tecidos, podendo inibir o crescimento tanto somático como ósseo.

Nos dias atuais, o maior desafio relativo à biologia do estresse é desenvolver um método de mensuração que possa ser diagnosticado clinicamente. A causa deste insucesso se relaciona principalmente a dois fatores: 1) o organismo animal nem sempre consegue manter a sua homeostase frente a um estímulo estressante por não ocorrer a ativação de seus sistemas de defesa; 2) cada estímulo estressante não desencadeia uma resposta específica do organismo (Koolhaas et al., 1999).

Outro desafio que também pode ser considerado está relacionado à variabilidade dos animais. Cada indivíduo poderá utilizar ferramentas biológicas diferentes frente a uma mesma situação e/ou intensidade de estresse. Isto ocorre porque há uma variedade de fatores intervenientes, tais como genética, idade, condição fisiológica, relações sociais, experiência antecipada e interações ser humanoanimal. Estes fatores modificam e reordenam a resposta individual frente ao estímulo estressante (Moberg, 2000).

Apesar das dificuldades de diagnóstico de um quadro de estresse, sabe-se, no entanto, que a primeira resposta biológica desencadeada pelo organismo, e indubitavelmente também a mais econômica, é a do comportamento (Barnett e Hemsworth, 1990). O animal, em primeiro lugar, irá mudar seu comportamento frente a situações de estresse, buscando ser bem sucedido em evitar a ameaça potencial (Koolhaas et al., 1999).

Os parâmetros comportamentais são de especial interesse porque podem ser medidos facilmente e de forma não invasiva. Quando tratamos de cães, os tutores são as pessoas que melhor entendem e percebem as mudanças de comportamento de seu animal de estimação (Mariti et al., 2012). Metodologias utilizando questionários com tutores, visando entender padrões comportamentais de cães, já foram amplamente utilizadas (Rooney e Bradshaw, 2004; Marinelli et al., 2007; Gazzano et al., 2008), inclusive usando como base as emoções (Kerswell et al., 2009), contudo, questionários com foco específico nos fatores estressantes e sua possível relação com as mudanças comportamentais de cães ainda foram pouco explorados na literatura.

Desta forma, o presente estudo teve por objetivo investigar a possível presença de alguns fatores estressores no ambiente de convívio de cães por meio da aplicação de questionário aos tutores. 


\section{Materiais e métodos}

Para a realização desta pesquisa foi utilizada uma amostra de conveniência constituída por 50 cães atendidos de forma recorrente no Hospital Veterinário da Universidade Federal de Uberlândia (UFU), Uberlândia, MG, sendo selecionados cães cujos tutores concordaram em participar da pesquisa. Considerou-se um cão com atendimento recorrente quando o mesmo passou por duas ou mais consultas veterinárias em um período de seis meses, desconsiderando o retorno. Foram incluídos na pesquisa apenas cães que eram considerados animais de estimação e que dividiam o mesmo ambiente de convívio com os entrevistados.

Durante a anamnese, um questionário previamente testado e padronizado foi preenchido para cada animal. As perguntas propostas no questionário levaram em consideração os principais aspectos comportamentais do cão que estariam relacionados a estresse (conforme descrito por Beerda et al., 1997), além de contemplarem os principais fatores ambientais e sociais que poderiam funcionar como estressores dentro do ambiente de convívio do cão. Estudos comportamentais tanto de humanos como de animais indicam que a integração de medidas comportamentais e sociais é importante na modelagem das diferenças individuais de comportamento (Chrousus e Gold, 1992; Granger e Kivlighan, 2003).

Anteriormente ao início da pesquisa, o questionário passou pela avaliação de um grupo de cinco médicos veterinários, que realizaram exame do conteúdo com aprovação das terminologias, visando a facilidade de entendimento do público-alvo.

Para fins de coleta de dados, o questionário foi dividido em cinco sessões focadas nos possíveis fatores estressantes no ambiente de convivência do cão (Beerda et al., 1997) e em mudanças de comportamento perceptíveis pelo dono (Beerda et al., 1999a,b): 1) fatores ambientais: envolvendo a descrição do local de permanência do animal; se houve mudança recente de ambiente ou se houve alguma mudança de rotina do cão (redução de horas de lazer e/ou mudança na dieta, por exemplo); 2) fatores genéticos: acerca dos pais do animal e da raça; 3) fatores de estresse prévio: envolvendo situações de traumas recentes; 4) fatores sociais: introdução recente de outros animais no convívio (intra ou inter espécie); relações tutor-cão relacionadas aos cuidados com o animal, horas de lazer, treinamento, punição e repreensão de comportamentos indesejáveis; e, por último, 5) mudança de comportamento: animal prefere ficar mais isolado, possui medo exagerado (quando apresenta mais de um dos comportamentos de evitação, fuga ou comportamento agressivo perante determinado estímulo), tem resposta negativa frente à separação do dono.

Os dados foram coletados por dois médicos veterinários residentes do Hospital Veterinário da UFU submetidos a treinamento prévio por meio de estudo piloto, e o questionário foi aplicado em momento de anamnese. As respostas obtidas foram digitadas para um banco de dados criado por meio do software EpiInfo 6.04 e a respectiva frequência de cada variável foi dada em forma de porcentagem, apresentada em formato de tabelas (Tabelas 1 a 6).

\section{Resultados e discussão}

Os animais de estimação, principalmente os cães, são criados, muitas vezes, de acordo com a rotina de vida dos seres humanos, geralmente privandoos de seus hábitos naturais e de sua vida instintiva, ou seja, privando-os de comportamentos inatos que são dependentes ontogeneticamente e modulados pelo ambiente, como o comportamento social. Tais alterações acabam por refletir diretamente na sua qualidade de vida (Berzins, 2000) e funcionam, muitas vezes, como fatores estressantes, desencadeando baixa imunidade nos animais.

Diante disso, conhecer o perfil dos cães atendidos de forma recorrente na rotina clínia pode ser de grande valia para o médico veterinário, a fim de que o mesmo possa adotar estratégias para minimizar os fatores estressantes e tratar clinicamente melhor seus pacientes.

\section{Fatores ambientais estressores}

Com relação aos fatores ambientais que possam atuar como estressores em cães (Tabela 1), observouse que a maioria dos tutores $(n=19,38 \%)$ referiram manter seus animais dentro de casa e no quintal. 
No entanto, 4\% ( $\mathrm{n}=2)$ das respostas merecem destaque, uma vez que houve manutenção dos animais em constante confinamento, restritos ao espaço físico da garagem e canil.

Tabela 1 - Distribuição porcentual de fatores ambientais considerados estressores, de acordo com as respostas de tutores de cães atendidos de forma recorrente no Hospital Veterinário da Universidade Federal de Uberlândia

\begin{tabular}{lcc}
\hline Variável & Número (n) & Porcentagem (\%) \\
\hline Local onde o animal é mantido & & \\
Dentro de casa & 8 & 16 \\
No quintal & 11 & 22 \\
Casa e quintal & 26 & 52 \\
Canil & 1 & 2 \\
Chácara & 2 & 4 \\
Fazenda & 1 & 2 \\
Garagem & 1 & 2 \\
Deslocamento do animal & & \\
Frequente & 29 & 58 \\
Algumas vezes & 6 & 12 \\
Raramente & 6 & 12 \\
Nunca muda & 9 & 18 \\
Modificações no local de criação & & \\
Sim & 35 & 70 \\
Não & 15 & 30 \\
Caracterização do ambiente onde & & \\
0 animal vive & & \\
Arejado & 34 & 68 \\
Quente e barulhento & 3 & 6 \\
Arejado e barulhento & 6 & 12 \\
Outros tipos & 7 & 14 \\
\hline & & \\
\hline
\end{tabular}

De acordo com a própria natureza biológica e comportamental do cão, restringir um espaço para sua manutenção pode contribuir como fator estressante, uma vez que predispõe, complica e agrava distúrbios psicológicos (Kobelt et al., 2003). Restrições quanto ao espaço físico afetam o bemestar geral e o comportamento dos cães, e estes estão diretamente relacionados à área disponível para o animal (Beerda et al., 1999a). Há registros de casos mais graves de distúrbios comportamentais em cães com restrição severa de espaço (mantidos em canis e correntes) (Clark et al., 1991), induzindo compor-tamentos de ócio, constate manipulação do ambiente e movimentos repetitivos (Hetts et al., 1992).

Quando nos referimos ao espaço físico domiciliar, no entanto, a comparação com a restrição de espaço dentro de apartamentos não demonstrou relação com problemas comportamentais ou com problemas de saúde dos cães (Soares et al., 2010a).

Outro fator ambiental considerado pelos tutores como possível causa de estresse foi a frequente mudança de ambiente, presente em 58\% ( $\mathrm{n}=29)$ das respostas, sendo as mais citadas: banho, tosa e hotelzinho. Também foi relatado pelos tutores que o ambiente de convivência do animal nos últimos meses passou por alguma transformação $(70 \%$, $\mathrm{n}=35$ ). As principais modificações citadas foram: "animal era da rua e recentemente foi para casa", "mudança de casa para apartamento", "mudança de endereço", "mudança do local de permanência dentro da casa", "não tem mais acesso à rua", "casa passando por reformas" e "animal antes ficava preso e agora não".

Desta forma, pressupõe-se que a maioria dos animais $(70 \%, n=35)$, pacientes recorrentes do Hospital Veterinário da UFU, foram recentemente submetidos a novos ambientes, o que pode ter dificultado sua capacidade de adaptação e consequente acúmulo de experiências ansiogênicas.

Em estudo pioneiro, Hetts et al. (1992) observaram diferenças comportamentais (maior tempo em movimento, aumento no número de estereotipias e maior número e intensidade de vocalizações) associadas às condições de mudanças nas habitações dos cães. Beerda et al. (2000) também reportaram mudanças comportamentais significativas quando cães mudaram de ambiente, aumentado o nível de atividade locomotora e comportamento de farejar e urinar.

Além disso, há na literatura referências de que mudanças na rotina do animal são fatores desencadeantes de estresse (Beaver, 2001), estando também relacionados à Síndrome da Ansiedade da Separação (SAS) (Beaver, 2001; Schwartz, 2003; Moreira, 2011), podendo ser causa de problemas de saúde ou afetar o bem-estar dos cães (Seibert e Landsberg, 2008). 
Por último, 68\% $(\mathrm{n}=34)$ dos tutores classificaram o ambiente do animal como arejado, o que indica a presença de um possível fator protetor do estresse entre os cães avaliados, uma vez que um ambiente arejado possibilita ao animal condições bioclimáticas agradáveis para seu conforto térmico e consequente bem-estar (Ferreira e Sampaio, 2010). Considerou-se um ambiente como arejado quando este continha espaços expostos ao ar livre.

Destaca-se, no entanto, a alta porcentagem de ambientes barulhentos $(18 \%, \mathrm{n}=9)$, ou seja, quando o local estava sem isolamento acústico, com ruas ao redor consideradas movimentadas pelo tutor. Para o cão, a predominância de sons em altas intensidades tem um agravante maior porque sua adição, diferentemente da humana, é mais sensível às amplitudes e intensidades sonoras. Enquanto cães abrangem de 40 a $65 \mathrm{kHz}$, os seres humanos conseguem captar uma frequência máxima de som de $20 \mathrm{kHz}$. Os cães também são mais sensíveis a frequências baixas, suportando a faixa de 0,5 a 16 $\mathrm{kHz}$, o que seria $24 \mathrm{db}$ a menos que o suportado pelos seres humanos (Overall, 2013).

\section{Fatores genéticos estressores}

Conforme citado por Kendler et al. (1999), os fatores genéticos devem ser levados em consideração em uma avaliação de estresse em cães. Apesar disso, os achados obtidos nesta pesquisa (Tabela 2) mostraram que $60 \%(\mathrm{n}=30)$ dos tutores entrevistados não detinham informações sobre os pais de seu animal. E nos casos afirmativos, 12\% $(n=6)$ consideravam os pais estressados (agressivos, destruidores e agitados). Estes dados aventam a possibilidade de que os tutores de animais de companhia, embora adquiram seus animais para uma convivência próxima, não levam em conta os padrões paternos ao adquirir um cão, podendo ter como consequência a criação do animal em um ambiente inapropriado, o que, combinado com o fator genético, pode predispor ao estresse.

Observa-se que as raças mais frequentes entre os cães avaliados (Tabela 2) foram Poodle $(20 \%, n$ $=10)$, Shih Tzu (6\%, $\mathrm{n}=3)$ e Cooker Spanel $(6 \%, \mathrm{n}=$ 3) que, de acordo com Bamberger e Houpt (2006), podem ser raças com maior potencial de estresse, juntamente com Dálmatas e Pastores Alemães.
Para estes autores, os comportamentos de estresse mais citados foram agressividade e medo/fobias generalizadas (a maioria relacionada a sons).

Tabela 2 - Distribuição porcentual de fatores genéticos considerados estressores, de acordo com as respostas de tutores de cães atendidos de forma recorrente no Hospital Veterinário da Universidade Federal de Uberlândia

\begin{tabular}{|c|c|c|}
\hline Variável & Número (n) & Porcentagem (\%) \\
\hline \multicolumn{3}{|c|}{ Pais do animal são conhecidos? } \\
\hline Sim & 20 & 40 \\
\hline Não & 30 & 60 \\
\hline \multicolumn{3}{|c|}{ Pais do animal eram estressados? } \\
\hline Sim & 6 & 12 \\
\hline Não & 13 & 26 \\
\hline Não sabe informar & 31 & 62 \\
\hline \multicolumn{3}{|l|}{ Raça do animal } \\
\hline SRD (sem raça definida) & 21 & 42 \\
\hline Poodle & 10 & 20 \\
\hline Pinscher & 2 & 4 \\
\hline Shih Tzu & 3 & 6 \\
\hline Cocker Spaniel & 3 & 6 \\
\hline Dachshund & 2 & 4 \\
\hline Boxer & 2 & 4 \\
\hline Perdigueiro/Pointer & 2 & 4 \\
\hline Yorkshire Terrier & 1 & 2 \\
\hline Pastor Alemão & 1 & 2 \\
\hline Weimaraner & 1 & 2 \\
\hline Rottweiller & 1 & 2 \\
\hline Setter Irlandês & 1 & 2 \\
\hline
\end{tabular}

A preferência por certas raças de cães muda constantemente ao longo do tempo, e quando surge uma demanda súbita por uma raça específica, algumas características genéticas podem ter impacto negativo sobre a saúde dos animais, com alterações fenotípicas e defeitos associados à raça (Asher et al., 2009). 0 acasalamento para aparência não leva em conta a genética do temperamento do animal (Beaver, 2001). Sendo assim, antes de adquirir um animal de estimação, o recomendado por muitos especialistas de comportamento 
canino (Houpt, 2005) é que se faça uma avaliação dos hábitos de vida do tutor para escolher a raça canina com melhores chances de adaptação à rotina, baseando-se em conhecimentos prévios de temperamento e reatividade da raça.

\section{Fatores de estresse prévio}

Sabe-se que uma experiência traumática, dependendo de sua extensão e gravidade, pode ser desencadeadora de condições patológicas comportamentais como fobias e estresse póstraumático (Flannigan e Dodman, 2001). Neste sentido, esta pesquisa (Tabela 3) procurou observar a presença de alguma condição traumática prévia entre os cães avaliados, verificando que esta condição estava presente em $30 \%(n=15)$ dos cães. Dentre as situações citadas, as de maior frequência foram ferimentos graves (ataque por outro animal, atropelamento, quedas) e cirurgias, somando $24 \%$ $(\mathrm{n}=12)$ dos casos.

Tabela 3 - Ocorrência de fatores de estresse prévio, de acordo com as respostas dos tutores de cães atendidos de forma recorrente no Hospital Veterinário da Universidade Federal de Uberlândia

\begin{tabular}{lcc}
\hline Variável & Número (n) & Porcentagem (\%) \\
\hline Experiência traumática prévia & 15 & 30 \\
Sim & 35 & 70 \\
Não & & \\
Experiências citadas* & 3 & 6 \\
Ataque por outro animal & 2 & 4 \\
Atropelamento & 3 & 6 \\
Quedas & 4 & 8 \\
Cirurgia & 2 & 4 \\
Doenças traumáticas & 1 & 2 \\
Animal ficava preso no banheiro & 3 & \\
\hline
\end{tabular}

Nota: * Pergunta válida somente para os tutores que responderam que os seus cães tiveram uma experiência traumática prévia. Desta forma, as porcentagens foram calculadas em relação ao total de 30 respostas.

Dreschel (2010) menciona que após a ocorrência de eventos traumáticos, o cão pode manifestar uma ansiedade crônica que leva a um quadro de estresse crônico e, logo, a um estímulo excessivo do eixo hipotálamo-hipófise-adrenal, aumentando os níveis de cortisol. Especula-se que o excesso deste hormônio, com o tempo, pode alterar os mecanismos de divisão celular e levar à morte precoce das células, antecipando e agravando o aparecimento de diversas doenças relacionadas aos sistemas imune, endócrino e nervoso, podendo, inclusive, diminuir a expectativa de vida dos cães.

\section{Fatores sociais como estressores}

Uma das características marcantes da vida social de cães é o convívio multiespécies, sendo que os humanos são parte do grupo. No entanto, quando há uma desestabilidade deste grupo, os cães podem sofrer com o estabelecimento de uma nova hierarquia (Miklósi, 2014).

Schwartz (2003) cita que muitos cães que já possuem alguma predisposição à SAS podem começar a demonstrar sinais frente a leves modificações, tal como alterações de horário na rotina do tutor, recolocação em um novo ambiente ou, principalmente, em relação à adição de novos animais de estimação, aumentando o nível de estresse do animal. Na presente pesquisa, 16\% $(\mathrm{n}=8)$ dos cães avaliados tiveram recentemente mudanças em seu convívio, com a adição de um novo animal ao grupo (Tabela 4).

Dos indivíduos amostrados, 64\% (n = 32) referiram ter os cuidados necessários com o seu animal. Quando questionados sobre as horas de lazer dedicadas a seu cão, porém, 30\% $(n=15)$ afirmaram que houve diminuição destas horas e $10 \%(\mathrm{n}=5)$ relataram que o cão não tem horas de lazer (Tabela 4).

Rotinas de lazer envolvem caminhar com o cão em vias públicas (parques, praças, avenidas e ruas) e, ainda, iniciar uma interação positiva com o animal com o intuito de brincadeira. Tais atividades são prazerosas e promovem uma boa relação ser humano-cão, fazendo-se necessárias ao animal. Uma diminuição brusca destas atividades pode causar quadros depressivos, problemas comportamentais e até enfermidades (Rooney e Bradshaw, 2002).

Os cães (Canis lupus familiaris) desempenham o comportamento de brincadeira muito mais que 
seus antepassados selvagens, possivelmente em decorrência a um efeito da neotonia comportamental da domesticação (Driscoll et al., 2009). Animais de companhia, tanto cães como gatos, realizam o comportamento de brincadeira, porém com motivações bem diferentes. A brincadeira de gatos comumente é dirigida a objetos semelhantes a presas, sendo desenvolvidos comportamentos similares aos de caça (Hall, 1998). Para o cão doméstico, no entanto, é mais provável que a brincadeira envolva um parceiro, seja canino ou humano, e esta parece ter uma motivação predominantemente social (Rooney et al., 2000; Ward e Smuts, 2007; Pullen, 2011). Desta forma, a diminuição das horas de lazer do cão pode impactar negativamente seu bem-estar, principalmente por envolver um caráter social (Trisko, 2011).

Além da diminuição do lazer, tutores também relataram mudança na dieta do animal, presente em $28 \%(n=14)$ das respostas, sendo que as mudanças de marca de ração e de forma de alimentação (comida para ração) foram as mais citadas.

Para a maioria dos cães domésticos, a seleção do alimento fornecido pelos humanos é baseada em sua aparência, odor, sabor e textura (Bradshaw, 2006), porém, embora este viés sensorial possa predispor os cães a preferirem alguns alimentos a outros, estas preferências são modificadas de acordo com a experiência vivida.

Os cães alimentados com a mesma dieta por longos períodos geralmente rejeitam outras dietas (Ferrell, 1984; Griffin et al., 1984). Este comportamento é denominado "efeito da novidade", que ocasiona rejeição de alimentos aparentemente palatáveis, porém que nunca foram experimentados. Esta neofobia inicial pode perdurar por dias, semanas ou até causar rejeição completa e persistente ao alimento, mesmo em encontros subsequentes (Bradshaw, 1991). Desta forma, uma mudança brusca na alimentação caracteriza um fator estressante para o animal e, em alguns casos, pode até prejudicar a quantidade de alimento ingerido, levando a quadros de anemia, anorexia e deficiências vitamínicas (Maarschalkerweerd et al., 1997).

Em relação às atitudes dos tutores frente ao comportamento de seus cães, $64 \%(n=32)$ repreendem o cão quando este tem comportamentos indesejáveis ou não apropriados, tendo sido a cópula com objetos ou pessoas o mais citado. Vale ressaltar que $40 \%(n=20)$ destes tutores tinham cães castrados.

Os problemas comportamentais são a principal razão para a orquiectomia, sendo o comportamento sexual indesejado a razão mais comum. No entanto, nem todos os machos passam por uma mudança de comportamento após a castração, por ser este um comportamento aprendido (Maarschalkerweerd et al., 1997).

Uma das principais causas para o abandono de cães (Scarlett et al., 2002), os problemas comportamentais desempenham um papel importante na percepção de apego do dono (Serpell, 1996). Comportamentos indesejáveis tais como monta em objetos e/ou pessoas, eliminação inadequada, perturbação das pessoas no momento da alimentação, agressão direta a pessoas ou cães desconhecidos e comportamento de brincar persistente são os mais comuns relatados pelos tutores (Gazzano et al., 2008) e podem ser evitados quando os cães previamente passam por uma socialização quando filhotes.

Até 14 semanas pós-nascimento, sugere-se que o filhote seja exposto a uma grande variedade de estímulos para que possa conhecer elementos essenciais de seu ambiente e rotina, servindo como base para a sua formação comportamental futura e eliminando, na maioria das vezes, os comportamentos indesejados (Seksel, 1999). Gazzano et al. (2008) mostraram que cães cujos tutores receberam conselhos de um veterinário comportamentalista nas primeiras semanas de vida se comportaram de forma positiva em relação a comportamentos indesejáveis, diferentemente de cães cujos tutores não tiveram esta oportunidade (Cannas et al., 2018).

Observa-se que $46 \%(n=23)$ dos tutores já puniram ou punem seus animais por meio de agressão direta (tapas, chineladas, objetos lançados contra o cão), sendo que as principais situações que desencadearam tal comportamento foram: agressividade com pessoas e/ou animais, brigas com outros cães, saída para a rua, destruição de objetos, latido intenso e repetitivo, e micção em local inapropriado (Tabela 4). 
Agressão é definida como um comportamento ou intenção por parte de um indivíduo de ferir ou de infligir estimulação nociva a outro organismo (Moyer, 1968). Quando o ser humano inicia uma resposta agressiva de repreensão ao cão, este, por sua vez, também pode desencadear um comportamento agressivo de defesa em resposta ao estímulo iniciado pelo homem ou um comportamento submisso de isolamento e medo (Coren, 2004).

Em relação ao medo, 74\% $(n=37)$ dos tutores afirmaram observar tal comportamento em seu cão, sendo que $4 \%(n=2)$ citam vassouras, varas e chinelos como os objetos que impõe medo ao animal (Tabela 5), o que remete à possibilidade de o cão não ter medo do objeto em si, mas sim da punição do dono que o agride com tais materiais, reforçando a presença da punição doméstica como possível fator social estressor para cães.

Tabela 4 - Ocorrência de fatores sociais considerados estressores, de acordo com as respostas de tutores de cães atendidos de forma recorrente no Hospital Veterinário da Universidade Federal de Uberlândia

\begin{tabular}{lcc}
\hline Variável & Número (n) & Porcentagem (\%) \\
\hline Outro animal para convivência & & \\
\hline Sim & 8 & 16,0 \\
Não & 42 & 84,0 \\
Cuidados com o cão & & \\
Em excesso & 8 & 16,0 \\
Necessários & 32 & 64,0 \\
Não cuida o quanto gostaria & 10 & 20,0 \\
Horas de lazer do cão & & \\
Houve diminuição da frequência & 15 & 30,0 \\
Continuam como antes & 26 & 52,0 \\
Aumentou a frequência & 4 & 8,0 \\
O cão não tem lazer & 5 & 10,0 \\
Cão foi submetido a treinamento & & \\
Sim & 8 & 16,0 \\
Não & 42 & 84,0 \\
Já puniu o cão com severidade & & 48,0 \\
Sim & 23 & 6,0 \\
Não & 24 & \\
Não informou & 3 & \\
\hline
\end{tabular}

(continuação)

\begin{tabular}{llc}
\hline Situações em que houve punição* & & \\
Agressividade com outros animais & 2 & 17,3 \\
Sair para a rua & 3 & 13,0 \\
Brigar com outros animais & 3 & 13,0 \\
Estragar objetos & 7 & 30,4 \\
Latir muito & 3 & 13,0 \\
Urinar dentro de casa & 2 & 17,3 \\
Não informou & 3 & 13,0 \\
Repreensão a comportamentos & & \\
indesejáveis & & \\
Frequentemente & 19 & 38,0 \\
Às vezes & 13 & 26,0 \\
Nunca & 18 & 36,0 \\
Houve mudanças na dieta do & & \\
animal & & 7,0 \\
Sim & 14 & 7,1 \\
Não & 1 & 7,1 \\
Mudanças de dieta citadas** & 36 & 7,1 \\
Carne moída com maior frequência & 1 & \\
Come a comida do gato & 1 & \\
Dieta restrita para perda de peso & 0 & \\
Diminuição de comida caseira & 1 & \\
Diminuição de petiscos & & \\
Mudança de marca de ração & & \\
Mudança de leite para ração & & \\
Somente ração agora & & \\
Não informou & & \\
\hline
\end{tabular}

Nota: * Pergunta válida somente para os tutores que responderam que punem seus cães; sendo assim, as porcentagens foram realizadas em relação ao total de 23 cães. ${ }^{* *}$ Pergunta válida somente para os tutores que responderam ter mudado a dieta de seu cão, totalizando 14 animais. Desta forma, as porcentagens também consideraram este valor como total.

\section{Mudanças comportamentais}

O comportamento de um cão pode dizer muito sobre seu estado em um determinado momento, sendo muito utilizado como indicador de estresse (Beerda et al., 1998; Bodnariu, 2008). Segundo Margis et al. (2003), as respostas comportamentais básicas de um cão frente a um fator estressor são enfrentamento (agitação/agressividade), evitação (fuga/isolamento) e passividade (que muitas vezes leva ao colapso do animal de forma repentina). 
0 mecanismo de enfrentamento foi relatado por $34 \%(n=17)$ dos tutores, que observaram uma maior agitação do animal, principalmente tentando chamar atenção do dono. Já o mecanismo de evitação foi relatado por $60 \%(\mathrm{n}=30)$ dos tutores, que observaram nos últimos seis meses que seu cão tem buscado ficar mais isolado. Ainda, $70 \%$ dos tutores relataram que quando se separam de seu cão, este fica "quieto", "triste", "amuado", demonstrando forte ligação afetiva (Tabela 5).

Tabela 5 - Descrição das mudanças comportamentais dos cães encaminhados ao Hospital Veterinário da Universidade Federal de Uberlândia, percebidas pelos tutores, durante os últimos 6 meses

\begin{tabular}{lcc}
\hline Variável & Número & Porcentagem (\%) \\
\hline $\begin{array}{l}\text { Animal tem preferido ficar mais } \\
\text { isolado }\end{array}$ & & \\
Sim & 20 & 60,0 \\
Não & 30 & 40,0 \\
$\begin{array}{l}\text { Animal mudou de comportamento } \\
\text { tentando chamar mais atenção }\end{array}$ & & \\
Sim & 17 & 34,0 \\
Não & 33 & 66,0 \\
Reação do cão quando há & & \\
separação do dono & & \\
Agitado & 2 & 4,0 \\
Indiferente & 11 & 22,0 \\
Quieto, triste, amuado & 36 & 72,0 \\
Acompanha o dono, segue-0 & 1 & 2,0 \\
Já notou algum medo exagerado & & \\
em seu cão & & \\
Sim & 37 & 74,0 \\
Não & 13 & 26,0 \\
O animal sente medo de quê* & & \\
Fogos de artifício & 18 & 48,6 \\
Trovões e tempestades & 12 & 32,4 \\
Varas e vassouras & 2 & 5,4 \\
Outros & 5 & 13,5 \\
\hline
\end{tabular}

Nota: * Pergunta válida somente para os tutores que responderam que os seus cães têm medo exagerado. Desta forma, as porcentagens foram calculadas em relação ao total de 37 respostas.
Uma condição considerada necessária para a ocorrência da SAS é a hipervinculação (McCrave, 1991; King et al., 2000; Landsberg et al., 2004), traduzida na organização da rotina do cão em torno da figura de vínculo, desencadeando sinais de ansiedade ou desconforto sempre que essa figura se afasta. As manifestações desta síndrome podem levar os animais a quadros depressivos, caracterizados por inatividade, principalmente quando há o relato do tutor de que o cão dorme durante todo o tempo em que está sozinho (McCrave, 1991; Landsberg et al., 2004). Nos Estados Unidos, o diagnóstico de SAS é o terceiro problema comportamental mais registrado (Takeuchi et al., 2001), com ocorrência entre 14\% (Denenberg et al., 2005) e 40\% (Seksel e Lindeman, 2001) nas clínicas, porcentagens menores do que as encontradas no presente estudo $(72 \%, \mathrm{n}=36$; Tabela 5).

\section{Principais motivos de encaminhamento do cão ao hospital veterinário}

Uma boa descrição da sintomatologia clínica do animal durante a anamnese pode auxiliar o médico veterinário a ter um diagnóstico preciso. Desta forma, saber quais as principais causas de encaminhamento do cão faz parte da rotina de qualquer centro veterinário (Tabela 6). Muitas vezes, no entanto, a anamnese voltada a problemas comportamentais deixa de ser realizada.

A ocorrência de problemas comportamentais em cães tem implicações importantes em seu bem-estar, podendo ocasionar baixa imunidade do animal e ser fonte de enfermidades recorrentes (Soares et al., 2010b). O negligenciamento da condução de uma anamnese comportamental pode comprometer o diagnóstico final e contribuir para que a doença tenha um caráter crônico/recorrente, uma vez que os principais fatores causadores ou intervenientes são de cunho psicológico (Beaver, 2001).

No Brasil, há uma carência de dados epidemiológicos relativos a distúrbios de comportamento e estresse em cães; por isso, o presente trabalho constitui um estudo exploratório dos possíveis fatores estressantes presentes na rotina do cão, em virtude do grande impacto que causam na qualidade de vida dos cães e de seus tutores. 
Tabela 6 - Dados referentes ao motivo de encaminhamento do cão ao Hospital Veterinário da Universidade Federal de Uberlândia

\begin{tabular}{lcc}
\hline Motivos & Número de cães & Porcentagem (\%) \\
\hline Prevenção & 8 & 16 \\
Procedimentos & 8 & 16 \\
Cirurgias & 6 & 12 \\
Trauma & 3 & 6 \\
Neoplasia & 20 & 40 \\
Sintomatologia clínica & 3 & 6 \\
Cardiopatia & 2 & 4 \\
Problema respiratório & 0 & 0 \\
Total & 50 & 100 \\
\hline
\end{tabular}

\section{Conclusão}

Conclui-se que os tutores de animais podem fornecer informações importantes e válidas acerca de possíveis fatores estressantes em cães, sejam eles de cunho ambiental, genético, social ou comportamental, e que as informações coletadas pelo questionário se mostraram relevantes para auxiliar o médico veterinário no diagnóstico final.

O estresse em cães e seus decorrentes problemas comportamentais têm impacto na qualidade de vida do cão e podem ser desencadeadores de doenças, levando o animal a consultas recorrentes. Mudanças na rotina familiar, inserção de terapia comportamental (como a desensibilização e condicionamento operante) ou até mesmo utilização de medicamentos são alternativas que estão em constante crescimento na clínica veterinária.

\section{Referências}

Asher L, Diesel G, Summers JF, McGreevy PD, Collins LM. Inherited defects in pedigree dogs. Part 1: Disorders related to breed standards. Vet J. 2009; 182(3):402-11.

Bamberger M, Houpt KA. Signalment factors, comorbidity, and trends in behavior diagnoses in dogs: 1644 cases (1991-2001).JAm Vet Med Assoc. 2006; 229(10):1591-601.
Barnett JL, Hemsworth PH. The validity of physiological and behavioural measures of animal welfare. Appl Anim Behav Sci. 1990;25(1-2):177-87.

Beaver BV. Comportamento canino: um guia para veterinários. São Paulo: Roca; 2001. 425p.

Beerda B, Schilder MBH, van Hooff J, de Vries HW. Manifestations of chronic and acute stress in dogs. Appl Anim Behav Sci. 1997;52(3-4):307-19.

Beerda B, Schilder MBH, van Hoof JARAM, de Vries HW. Behavioral saliva cortisol and heart rate responses to different stimuli in dogs. Appl Anim Behav Sci. 1998;58(3-4):365-81.

Beerda B, Schilder MBH, van Hoof JARAM, de Vries HW, MOL JA. Chronic stress in dogs subjected to social and spatial restriction. I. Behavioral responses. Physiol Behav. 1999a;66(2):233-42.

Beerda B, Schilder MBH, Bernadina W, van Hoof JARAM, de Vries HW, MOL JA. Chronic stress in dogs subjected to social and spatial restriction. II. Hormonal and immunological responses responses. Physiol Behav. 1999b;66(2):243-54.

Beerda B, Schilder MBH, van Hoff JARAM, de Vries HW, Mol JA. Behavioural and hormonal indicators of enduring environmental stress in dogs. Anim Welf. 2000;9:49-62.

Berzins MAVS. Velhos, cães e gatos: interpretação de uma relação [dissertação]. São Paulo: Pontifícia Universidade Católica de São Paulo; 2000. 162 p.

Blecha F. Immune System response to stress. In: Moberg GP, Mench JA (EE.). The biology of animal stress: basic principles and implications for animal welfare. New York: CABI Publishing; 2000. p. 111-22.

Bodnariu A. Indicators of stress and stress assessment in dogs. Lucr Stiint Med Vet. 2008;41:20-6.

Bradshaw JW. Sensory and experiential factors in the design of food for domestic dogs and cats. Proc Nutr Soc. 1991;50(1):99-106. 
Bradshaw JW. The evolutionary basis for the feeding behavior of domestic dogs (Canis familiaris) and cats (Felis catus). J Nutr. 2006;136(Suppl 7):1927S-1931S.

Cannas S, Talamonti Z, Mazzola S, Minero M, Picciolini A, Paestrini C. Factors associated with dog behavioral problems referred to a behavior clinic. J Vet Behav. 2018;24:42-7.

Chrousus GP, Gold PW. The concepts of stress and stress system disorders. JAMA. 1992;267(9):1244-52.

Clark JD, Calpin JP, Armstrong RB. Influence of type of enclosure on exercise fitness of dogs. Am J Vet Res. 1991;52(7):1024-8.

Coren S. How dogs think - understanding the canine mind. New York: Free Press; 2004. 368 p.

Denenberg S, Landsberg GM, Horwitz D, Seksel K. A comparison of cases referred to behaviorists in three different countries. In: Mills D et al. (EE.). Current issues and research in veterinary behavioral medicine. Indiana: Purdue University Publications; 2005. p. 56-62.

Dreschel NA. The effects of fear and anxiety on health and lifespan in pet dogs. Appl Anim Behav Sci. 2010;125(3-4):157-62.

Driscoll CA, Macdonald DW, O’Brien SJ. From wild animals to domestic pets, an evolutionary view of domestication. Proc Natl Acad Sci U S A. 2009;106(Suppl 1):9971-8.

Ferreira SA, Sampaio IBM. Relação homem-animal e bemestar do cão domiciliado. Arch Vet Sci. 2010;15:(1)22-35.

Ferrell F. Effects of restricted dietary flavor experience before weaning on postweaning food preference in puppies. Neurosci Biobehav Rev. 1984;8(2):191-8.

Flannigan G, Dodman NH. Risk factors and behaviors associated with separation anxiety in dogs. J Am Vet Med Assoc. 2001;219(4):460-6.

Gazzano A, Mariti C, Alvares S, Cozzi A, Tognetti R, Sighieri C. The prevention of undesirable behaviors in dogs: effectiveness of veterinary behaviorists' advice given to puppy owners. J Vet Behav. 2008;3(3):125-33.
Granger DA, Kivlighan KT. Integrating biological, behavior and social levels of analysis in early child development research: progress, problems, and prospects. Child Dev. 2003;74(4):1058-63.

Griffin RW, Scott GC, Cante CJ. Food preferences of dogs housed in testing-kennels and in consumers'homes: some comparisions. Neurosci Biobehav Rev.1984; 8(2):253-9.

Hall SL. Object play by adult animals. In: Bekoff M, Byers JA (EE). Animal Play: Evolutionary, Comparative and Ecological Perspectives. New York: Cambridge University Press; 1998. p. 45-60.

Hetts S, Clark JD, Calpin JP, Arnold CE, Mateo JM. Influence of housing conditions on beagle behavior. Appl Anim Behav Sci. 1992;34(1-2):137-55.

Houpt KA. Domestic animal behavior for veterinarians and animal scientists. Iowa: Blackwell Publishing; 2005. $333 \mathrm{p}$.

Kendler KS, Karkowski LM, Prescott CA. Causal relationship between stressful life events and the onset of major depression. Am J Psychiatry.1999;156(6): 837-41.

Kerswell KJ, Bennett PJ, Butler KL, Hemsworth PH. Selfreported comprehension ratings of dog behavior by puppy owners. Anthrozoos. 2009;22(2):183-93.

King JN, Simpson BS, Overall KL, Appleby D, Pageat $\mathrm{P}$, Ross C, et al. Treatment of separation anxiety in dogs with clomipramine: results from a prospective, randomized, double-blind, placebo-controlled, parallelgroup, multicenter clinical trial. Appl Anim Behav Sci. 2000;67(4):255-75.

Kobelt AJ, Hemsworth PH, Barnett JL, Butler KL. Sources of sampling variation in saliva cortisol in dogs. Res Vet Sci. 2003;75(2):157-61.

Koolhaas JM, Korte SM, De Boer SF, Van Der Vegt BJ, Van Reenen CG, Hopster $\mathrm{H}$, et al. Coping styles in animals: current status in behavior and stress-physiology. Neurosci Biobehav Rev. 1999;23(7):925-35. 
Landsberg GM, Hunthausen WL, Ackerman L. Problemas comportamentais do cão e do gato. São Paulo: Roca; 2004. 492 p.

Maarschalkerweerd RJ, Endenburg N, Kirpensteijn J, Knol BW. Influence of orchiectomy on canine behaviour. Vet Rec. 1997;140(24):617-9.

Margis R, Picon P, Cosner AF, Silveira RO. Relação entre estressores, estresse e ansiedade. Rev Psiquiatr do Rio Gd Sul. 2003;25(Suppl.1):65-74.

Marinelli L, Adamelli S, Normando S, Bono G. Quality of life of the pet dog: influence of owner and dog's characteristics. Appl Anim Behav Sci. 2007;108(1-2):143-56.

Mariti C, Gazzano A, Moore JL, Baragli P, Chelli L, Sighieri C. Perception of dogs' stress by their owners. J Vet Behav. 2012;7(4):213-9.

Mccrave EA. Diagnostic criteria for sepration anxiety in the dog. Vet Clin North Am Small Anim Pract 1991;21(2):247-55.

Miklósi A. Dog behaviour, evolution and cognition. Lexingon: Oxford University Press; 2014. 400 p.

Moberg GP. Biological response to stress: implications for animal welfare. In: Moberg GP, Mench JA (EE.). The biology of animal stress: basic principles and implications for animal welfare. New York: CABI Publishing; 2000. p.1-22.

Moreira HICD. Problemas comportamentais nos animais de companhia [dissertação]. Lisboa: Universidade Técnica de Lisboa; 2011. 123 p.

Moyer KE. Kinds of aggression and their physiological basis. Commun Behav Biol. 1968;2(2):65-87.

Overall KL. Manual of Clinical Behavioral Medicine for Dogs and Cats. St. Louis: Elsevier; 2013. 797 p.

Pullen AJ. Behavioural indicators of candidate enrichment for kennel housed dogs [tese]. Bristol: University of Bristol; 2011. 258 p.
Rensis FD, Scaramuzzi JR. Heat Stress and seasonal effects on reproduction in the dairy cow: a review. Theriogenology. 2003;60(6):1139-51.

Rooney N, Gaines S, Hiby E. A practitioner's guide to working dog welfare. J Vet Behav. 2009;4(3):127-34.

Rooney NJ, Bradshaw JWS, Robinson IH. A comparison of dog-dog and dog-human play behavior. Appl Anim Behav Sci. 2000; 66(3):235-48.

Rooney NJ, Bradshaw JWS. An experimental study of the effects of play upon the dog-human relationship. Appl Anim Behav Sci. 2002;75(2):161-76.

Rooney NJ, Bradshaw JWS. Breed and sex differences in the behavioral attributes of specialist search dogs - a questionnaire survey of trainers and handlers. Appl Anim Behav Sci. 2004;86(1-2):123-35.

Scarlett JM, Salman MD, New JG, Kass PH. The role of veterinary practioners in reducing dog and cat relinquishments and euthanasias. J Am Vet Med Assoc. 2002; 220(3):306-11.

Schwartz S. Separation anxiety syndrome in dogs and cats. J Am Vet Med Assoc. 2003;222(11):1526-32.

Seibert LM, Landsberg GM. Diagnosis and management of patients presenting with behavior problems. Vet Clin North Am Small Anim Pract. 2008;38(5):937-50.

Seksel K. Puppy socialization classes. Vet Clin North Am Small Anim Pract. 1999; 27(3):465-77.

Seksel K, Lindeman MJ. Use of clomipramine in treatment of obsessive-compulsive disorder, separation anxiety and noise phobia in dogs: a preliminary, clinical study. Aust Vet J. 2001;79(4):252-6.

Serpell JA. Evidence for an association between pet behavior and owner attachment levels. Appl Anim Behav Sci. 1996;47(1-2):49-60.

Soares GM, Pereira JT, Paixão RL. Estudo exploratório da síndrome de ansiedade de separação em cães de apartamento. Cienc Rural. 2010a;40(3):548-53. 
Soares GM, Souza-Dantas LM, D’Almeida JM, Paixão RL. Epidemiologia de problemas comportamentais em cães no Brasil: inquérito entre médicos veterinários de pequenos animais. Cienc Rural. 2010b;40(4):873-9.

Takeuchi Y, Ogata N, Houpt KA, Scarlett JM. Differences in background and outcome of three behavior problems of dogs. Appl Ani Behav Sci. 2001;70(4):297-308.

Trisko RK. Dominance, Egalitarianism and friendship at a dog day care facility [tese] Michigan: University of Michigan; 2011. 141 p.

Ward C, Smuts BB. Why does carnivore play matter? J Dev Process. 2007;2(2):31-8. 